\title{
Coherent combining of the output of two semiconductor lasers using optical phase-lock loops
}

\author{
Wei Liang and Amnon Yariv \\ Department of Applied Physics, MS 128-95, California Institute of Technology, Pasadena, California 91125, USA
}

Anthony Kewitsch and George Rakuljic

Telaris Inc., 2118 Wilshire Boulevard No. 238, Santa Monica, California 90403, USA

Received August 29, 2006; revised November 9, 2006; accepted November 10, 2006; posted November 14, 2006 (Doc. ID 74481); published January 26, 2007

\begin{abstract}
We have experimentally demonstrated current-injection optical phase-lock loops (OPLLs) based on commercial single-section semiconductor distributed-feedback (DFB) lasers. Using two parallel OPLLs, we have obtained $87 \%$ efficient coherent power combining of the two DFB lasers. The rms differential phase error between the two lasers is about $30^{\circ}$. (C) 2007 Optical Society of America

OCIS codes: $140.2010,030.1640,140.3490$.
\end{abstract}

High-power high-brightness laser systems made by coherently combining a large number of light emitters are of great interest to a range of applications that includes material processing, remote sensing, and precompensation and adaptation in atomospheric beam propagation. ${ }^{1,2}$ Various technologies have been explored to achieve the goal by using semiconductor (SC) lasers and fiber lasers. ${ }^{1,3-8}$ In this Letter we report what we believe to be the first coherent power addition of two commercial grade SC lasers by using current-injection optical phase-lock loops ${ }^{9-13}$ (OPLLs). Compared with the previous coherent power-combining techniques, including the optical injection OPLLs, the current-injection OPLLs technique can provide individual electronic control of the frequency and phase of each laser in an array without external phase modulators and thus can lead to a big coherent emitting aperture that can be controlled, focused, distortion corrected, scanned in space, and pulsed by using pure electronic control. Throughout the rest of this Letter we will use "OPLLs" to refer to the current injection OPLLs only.

In an OPLL, the phase and frequency of the slave laser are forced to track those of the master laser by a negative feedback control loop. Ever since the early pioneering work and demonstration of the first OPLL, ${ }^{9}$ different types of lasers have been used to build OPLLs. ${ }^{10-13}$ Compared with fiber lasers and solid state lasers, SC lasers are especially attractive for applications requiring high power and high efficiency. In addition, the frequency and phase control of SC lasers can be conveniently realized by control of the injection current. Implementing OPLLs based on SC lasers, however, has proved to be a challenging task, since, because of the large laser linewidth, it requires wideband feedback electronics and small feedback loop delays. Unfortunately, the achievable loop bandwidth is seriously limited, as single-section SC lasers typically exhibit a phase reversal in their FM response in the frequency range of $0.1-10 \mathrm{MHz}$ due to the competition between thermal and electronic effects. ${ }^{14,15}$ This makes it very difficult, if not impossible, to lock single-section SC lasers with a linewidth of above a few megahertz. Recently, with improvements in the design and the fabrication process, the linewidth of SC lasers has been reduced to submegahertz, which makes it possible to phase lock them with just a few megahertz of loop bandwidth. In our experiment, we successfully phase locked commercial distributed-feedback (DFB) lasers of fractional megahertz linewidth.

To determine the current frequency response of the free-running SC laser, we employed a fiber MachZehnder interferometer as a frequency discriminator and used an Agilent 4395A network analyzer. The details of the measurement procedure are similar to those described in Ref. 16. In our measurement we biased the DFB laser at $400 \mathrm{~mA}$, yielding an output power of $16 \mathrm{dBm}$ and a wavelength of $1538 \mathrm{~nm}$. The measured FM response of the laser is plotted in Fig. 1. Near $5 \mathrm{MHz}$, the amplitude of the FM response manifests a dip, and the phase of the FM response exhibits a $90^{\circ}$ shift. Added to the $90^{\circ}$ shift due to the inherent integration by the laser, ${ }^{2}$ and the $180^{\circ}$ shift

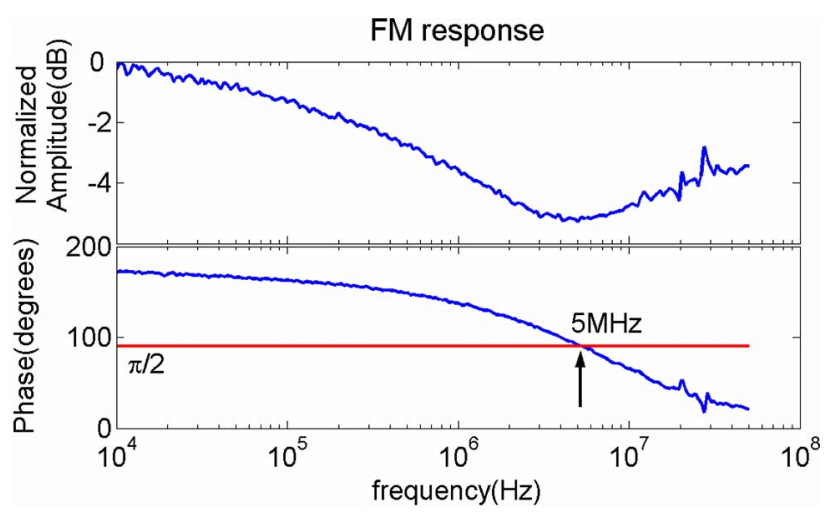

Fig. 1. (Color online) Measured FM response of a singlesection DFB laser. 


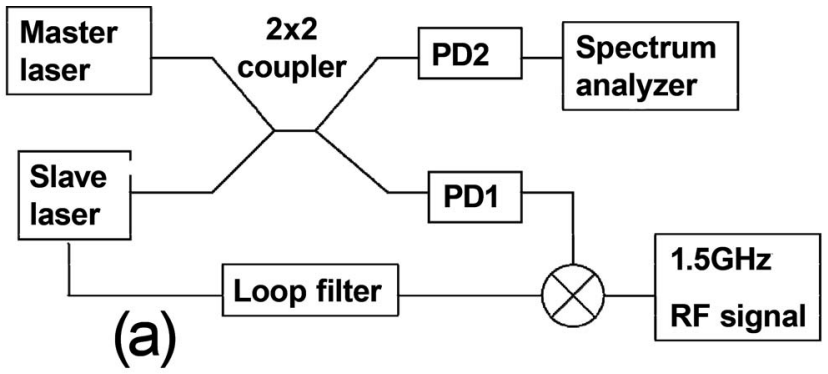

power spectrum of locked beat signal

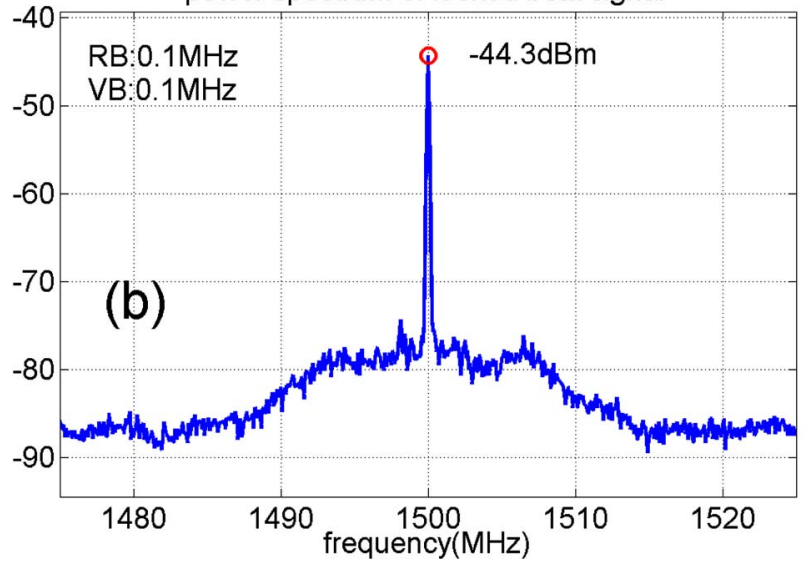

Fig. 2. (Color online) (a) Schematic of an OPLL. (b) Measured power spectrum of a $1.5 \mathrm{GHz}$ locked beat signal.

due to the negative feedback loop, the resulting $2 \pi$ phase shift near $5 \mathrm{MHz}$ combined with sufficient loop gain can cause oscillation and instability in the feedback loop. In this simple analysis we neglect the effect of the feedback time delay, an assumption that will be justified later.

Figure 2(a) is a schematic plot of one of the two OPLLs. We used an Agilent 81640A tunable laser with a submegahertz linewidth as the master laser. The optical signals from both lasers are combined by using a $2 \times 2$ optical fiber coupler. Half of the combined signal is fed to a HP 11982A photodetector (PD), and the resulting beat signal is fed to a HP $8565 \mathrm{E}$ rf spectrum analyzer. The beat signal output of the second PD (New Focus 1544B) is mixed with the $1.5 \mathrm{GHz}$ offset $\mathrm{rf}$ signal generated by a HP $8350 \mathrm{~A}$ signal generator. The mixer acts as a phase detector. The phase error signal passes through a loop filter and is then injected into the DFB laser to complete the control loop. In our experiment we used a firstorder lead-lag filter, which can increase the signal-tonoise ratio of the OPLL. We estimate the total loop delay time as $\sim 5 \mathrm{~ns}$. At $5 \mathrm{MHz}$ this corresponds to a $9^{\circ}$ phase delay, which is not significant in limiting the loop bandwidth. The acquisition bandwidth is measured to be about $10 \mathrm{MHz}$; the frequency difference between the two lasers needs to be manually tuned to the vicinity of the offset frequency $1.5 \mathrm{GHz}$ for locking to occur. In our next design this function will be performed automatically. The power spectrum of the beat signal between the master and the slave lasers is plotted in Fig. 2(b). The strength of the locked signal is more than $30 \mathrm{~dB}$ above the noise pedestal. From the spectrum, we can calculate the locked signal power and the noise power within the $50 \mathrm{MHz}$ sweep bandwidth. We estimate $90 \%$ optical power of the DFB laser is phase locked to the master laser. The residual rms differential phase noise between the two lasers is estimated to be $19^{\circ}$.

In a second experiment we phase locked two DFB slave lasers separately to a single (Agilent 81640A) master laser and combined their output. The schematic of the experimental setup is illustrated in Fig. $3(\mathrm{a})$. The two OPLLs are offset by the same $1.5 \mathrm{GHz}$ rf signal. The optical signals from the two OPLLs are combined by using a $2 \times 2$ optical fiber coupler. One output is used to simultaneously monitor the locking status of the two slave lasers to the master laser in the frequency domain by using the HP $8565 \mathrm{E}$ spectrum analyzer. The second output is displayed "as is" in the time domain using a Tektronix TDS3052B oscilloscope. The latter beat signal is shown in Fig. 3(b). If we ignore the slight power difference between the combined optical signals, the combined power at the $\mathrm{PD}$ is

$$
P=2 I\left[1+\cos \left(\Delta \omega t+\Delta \phi+\phi_{n}\right)\right]
$$

where $I$ stands for the power of each of the two optical signals, and $\Delta \omega, \Delta \phi$, and $\phi_{n}$ are respectively the frequency difference, phase difference, and differential phase noise between the combined two optical
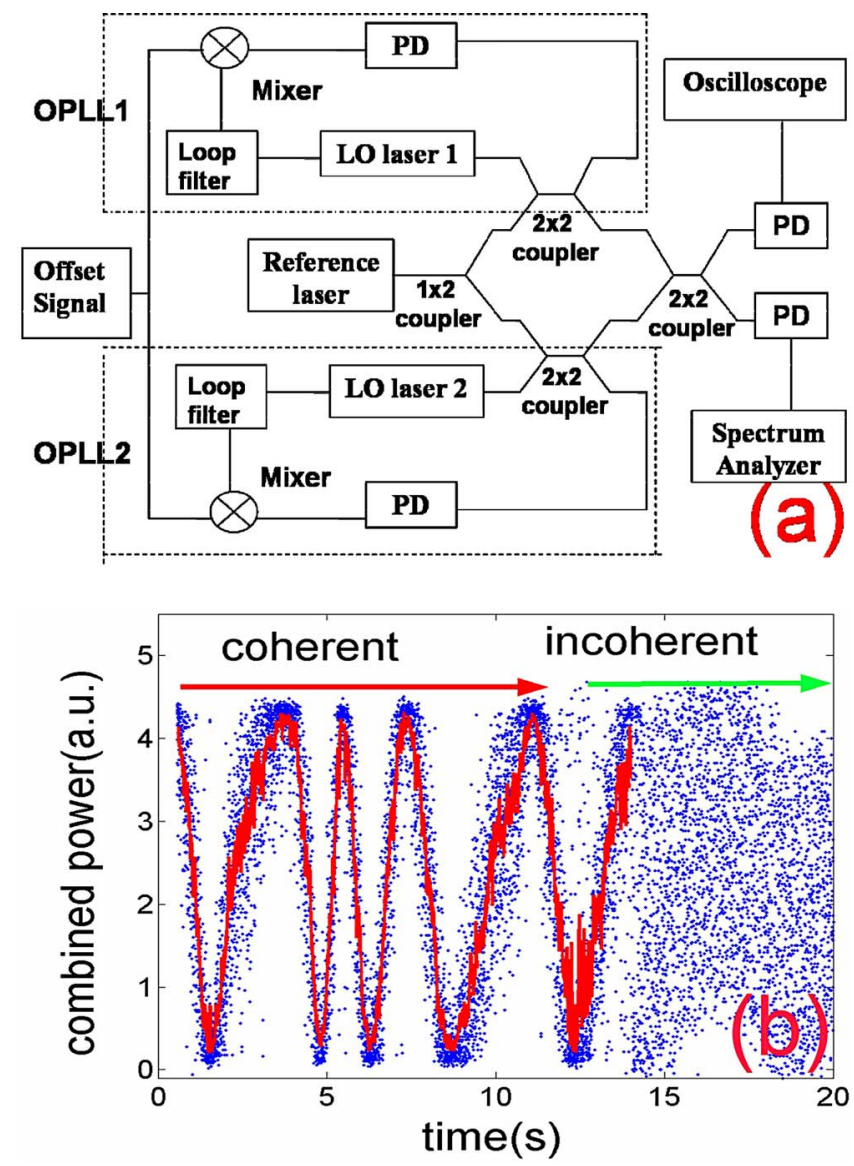

Fig. 3. (Color online) (a) Experimental setup for coherent power addition of two SC DFB lasers locked to a common reference laser. (b) Time domain measurement of combined power. Blue dots, measured data; red solid curve, smoothed data. 
signals at the PD. When at least one of the two slave lasers is not locked to the reference laser, the output is an ac signal [the right part of Fig. 3(b)] with the frequency of $\Delta \omega$. The data show as a noisy scatter of points since $\Delta \omega$ is in the megahertz range, while the time scale of the oscilloscope is set at seconds. When both DFB lasers are locked $(\Delta \omega=0)$, the signals are coherently added and the output of the PD consists of, ideally, a dc signal that in our case varies slowly on the time scale of seconds as can be seen in the left part of Fig. 3(b) (blue dots). This slow variation reflects, as it is should, the change of the difference in optical path lengths experienced by the two combined optical signals due to slow variation of temperature and environment. The variation of the optical path lengths will be significantly reduced as we move to free-space power combining later. Since the optical phase of the slave laser output signal can be directly controlled by the feedback current signal, the slow phase variation can also be compensated with an additional feedback loop made of optical phase detectors and electric phase shifters. By calculating the contrast, i.e., $\left(P_{\max }-P_{\min }\right) /\left(P_{\max }+P_{\min }\right)$, of the smoothed data [red solid curve in Fig. 3(b)], we estimate the beam combining efficiency is about $87 \%$. From the uncertainty of the dc signal (i.e., the scattering of the data) we can estimate the rms phase error $\phi_{n}$ between the two combined signals is about $30^{\circ}$. In the first experiment described above we have estimated from the power spectrum that the rms phase error between the slave laser and the master laser in each OPLL is about $19^{\circ}$; thus the rms phase error between the two slave lasers can be approximated as $\sqrt{2} \times 19=27^{\circ}$. Thus the rms phase errors calculated from the frequency domain and that from the time domain measurements agree with each other. In the above measurement and analysis, the contribution of the master laser in the combined output power is negligible, which can be justified by the fact that its output power is almost $16 \mathrm{~dB}$ smaller than those of the slave lasers.

In conclusion, we have coherently added the optical power of two SC DFB lasers by phase locking them to a single master laser by using OPLL technology. This points the way to potential coherent combining of a large array of SC lasers. Further improvement in the locking efficiency requires either that the laser linewidth be further reduced or that the loop bandwidth be increased. Efforts along these directions are in progress.

The authors acknowledge the support of DARPA's Microsystems Technology Office office (M. Stickley). W. Liang's e-mail address is liangwei@its.caltech.edu.

\section{References}

1. A. Shirakawa, T. Saitou, T. Sekiguchi, and K. Ueda, Opt. Express 10, 1167 (2002).

2. A. Yariv, Opt. Lett. 30, 2191 (2005).

3. N. W. Carlson, G. A. Evans, J. M. Hammer, M. Lurie, S. L. Palfrey, and A. Dholakia, Appl. Phys. Lett. 50, 1301 (1987).

4. J. R. Leger, M. L. Scott, and W. B. Veldkamp, Appl. Phys. Lett. 52, 1771 (1988).

5. W. Wang, K. Nakagawa, S. Sayama, and M. Ohtsu, Opt. Lett. 17, 1593 (1992).

6. V. A. Kozlov, J. Hernandez-Cordero, and T. F. Morse, Opt. Lett. 24, 1814 (1999).

7. L. Bartelt-Berger, U. Brauch, A. Giesen, H. Huegel, and H. Opower, Appl. Opt. 38, 5752 (1999).

8. S. J. Augst, T. Y. Fan, and A. Sanchez, Opt. Lett. 29, $474(2004)$.

9. L. H. Enloe and J. L. Rodda, Proc. IEEE 53, pp. 165 (1965).

10. W. R. Leeb, H. K. Philipp, A. L. Scholtz, and E. Bonek, Appl. Phys. Lett. 41, 592 (1982).

11. R. C. Steele, Electron. Lett. 19, 69 (1983).

12. T. J. Kane and E. A. P. Cheng, Opt. Lett. 13, 970 (1988).

13. L. N. Langley, M. D. Elkin, C. Edge, M. J. Wale, U. Gliese, X. Huang, and A. J. Seeds, IEEE Trans. Microwave Theory Tech. 47, 1257 (1999).

14. S. Kobayashi, Y. Yamamoto, M. Ito, and T. Kimura, IEEE J. Quantum Electron. 18, 582 (1982).

15. P. Correc, O. Girard, and I. F. de Faria, Jr., IEEE J. Quantum Electron. 30, 2485 (1994).

16. W. V. Sorin, K. W. Chang, G. A. Conrad, and P. R. Hernday, J. Lightwave Technol. 10, 787 (1992). 